\title{
Artelogie
}

Recherche sur les arts, le patrimoine et la littérature de l'Amérique latine

1 | 2011

Brésil, questions sur le modernisme

\section{Roberto Burle Marx dans le chaudron moderniste brésilien : la part du paysage}

Jacques Leenhardt

\section{OpenEdition \\ Journals}

Édition électronique

URL : https://journals.openedition.org/artelogie/8457

DOI : 10.4000/artelogie.8457

ISSN : 2115-6395

\section{Éditeur}

Association ESCAL

\section{Référence électronique}

Jacques Leenhardt, «Roberto Burle Marx dans le chaudron moderniste brésilien : la part du paysage », Artelogie [En ligne], 1 | 2011, mis en ligne le 01 mars 2011, consulté le 07 janvier 2022. URL : http:// journals.openedition.org/artelogie/8457 ; DOI : https://doi.org/10.4000/artelogie.8457

Ce document a été généré automatiquement le 7 janvier 2022.

Association ESCAL 


\title{
Roberto Burle Marx dans le chaudron moderniste brésilien : la part du paysage
}

\author{
Jacques Leenhardt
}

1 L'image d'un chaudron moderniste renvoie à l'idée d'effervescence active. Elle place d'emblée l'artiste et le poète du côté de l'action plutôt que de la contemplation, de la construction plutôt que de la provocation, du mélange et du partage plutôt que de la voie solipsiste. Cette métaphore convient donc particulièrement aux années de transition entre les années d'essoufflement de la Republiqua Velha (1889-1930) et l'avènement de l'Estado novo (1937), période où valeurs et alliances se transforment et où le rôle des artistes semble prendre un tour nouveau.

2 Cette exigence de renouveau est énoncée par Mario de Andrade, pour qui il convient de distinguer deux périodes du modernisme brésilien. Une première désignerait l'activité agitée et désorganisée du début des années 20 , avec comme point culminant la fameuse Semaine d'art moderne de 1922 à São Paulo, tandis que l'autre se caractériserait par un engagement plus réfléchi dans l'action, sociale aussi bien que politique et se développerait tout au long de la décennie et au-delà. Il ne s'agit pas d'abord d'un engagement partisan mais plutôt de la conscience insistante que l'activité artistique doit abandonner les chimères anciennes d'une «BEAUTE » importée. La modernité est un souci neuf de transformation intégrale de la conscience de soi, de la sensibilité et donc de la psychologie d'un peuple tout entier. 


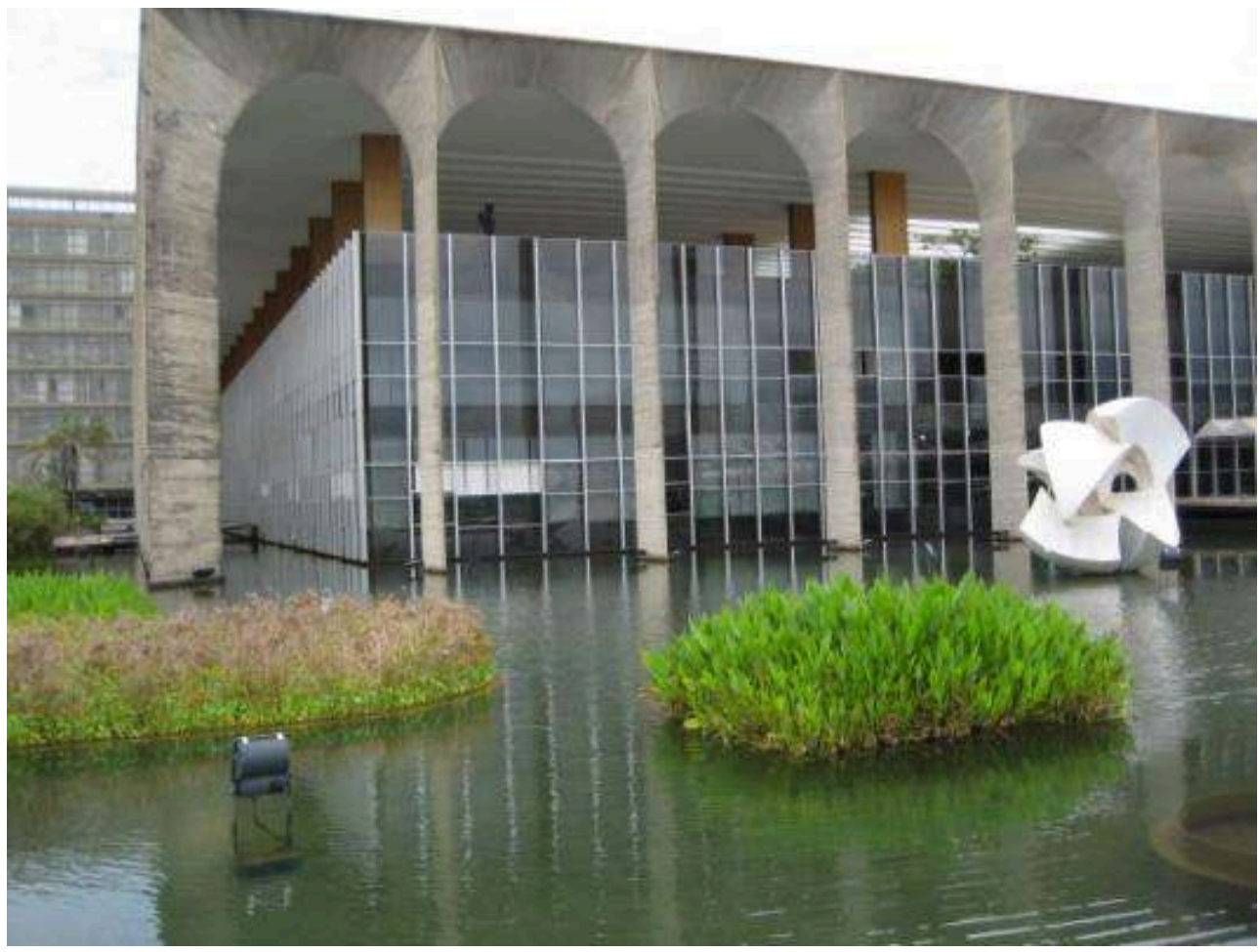

Cette entreprise à bien des égards pratique vise à faire advenir une espèce humaine encore inconnue, introuvable aux yeux de Mario de Andrade : le Brésilien. «Le Brésil a tout : la sécheresse du Céara, la maladie du café, la lèpre, la politique, le barbier, des patriotes (?), des belles femmes, la baie de Guanabara, des révolutions incapables de triompher et l'Amazone. Il a même des poètes, bon Dieu. Il lui manque simplement des Brésiliens. Même la lanterne de Diogène ne saurait en trouver. ${ }^{1}$

4 L'actualité artistique de la décennie précédente se détache encore sur un fond vieilli de poésie parnassienne que le «futurisme » de Marinetti a bousculé, sur une galerie non moins rance de peintures académiques et, en architecture, sur des styles vernaculaires ou éclectiques fleurissant sur les dépouilles d'un néo-classicisme d'importation désormais caduc.

La modernisation du Brésil s'était faite en trois étapes. La première était fondée sur les puissants maîtres latifondiaires du nord est, enrichis par l'esclavage et le sucre. La seconde reposait sur le caoutchouc (latex extrait de l'Hevea Brasiliensis) recueilli par les seringueiros au hasard de la forêt amazonienne, dont la splendeur ne dura que le temps pour d'habiles concurrents de développer des plantations d'hevea à Ceylan et dans le reste de l'Asie sous influence britannique, puis pour les chimistes d'inventer un substitut de synthèse. Enfin ce fut l'essor du café dont quelques barons furent, avec d'autres entrepreneurs, à l'origine de la révolution culturelle moderniste des années 20.

6 La République, qui a succédé à l'Empire se trouve alors soumises à une forte concentration urbaine provoquée par l'essor industriel qui marginalise l'ancienne classe des propriétaires fonciers. Une bourgeoisie se développe tandis que des alliances politiques nouvelles redessinent les rapports entre l'élite du pouvoir et les masses, et transforment la ville et les rapports sociaux. Un Brésil nouveau est en gestation, qui se cherche une identité post coloniale et post impériale. 
7 La Révolution de 1930, qui mettra fin à la «Republica Velha» marque un point tournant dans ce processus avec la venue au pouvoir de Getúlio Vargas, un positiviste convaincu dont le pouvoir allait bientôt se faire de plus en plus dictatorial. Par étapes, depuis une décennie, les thèmes de la modernisation industrielle et urbaine se mêlent à ceux de l'identité culturelle dans une dynamique commune. ${ }^{2}$ On le verra dans l'affaire du concours d'architecture pour le Ministère de l'Education et de la Santé publique (MES). Le jury avait retenu un projet traditionnaliste. Le milieu intellectuel moderniste se mobilisa alors pour suggérer au Ministre Capanema de réunir une équipe d'architectes favorables aux nouvelles techniques constructives et à l'esthétique moderne.

8 Les idées modernistes en architecture avaient lentement fait leur chemin. Gregory Warchavchik avait publié dès 1925 « Acerca da arquitetura moderna » où il combattait les préjugés stylistiques, les décorations aberrantes et plaidait pour une architecture logique et une beauté fonctionnelle. Il s'associera ensuite à Lúcio Costa pour un projet de maisons ouvrières auquel participera également Oscar Niemeyer (Gamboa, Rio de Janeiro, 1934) tandis que Affonso Eduardo Reidy, Júlio de Abreu et Rino Levi, arrivé d'Italie en 1928, développaient de leur côté une œuvre de pure inspiration moderniste. L'enseignement de Lúcio Costa à l'Ecole Nationale des beaux-arts (ENBA) et l'exposition dont il fut responsable au Salon de 31 divulguèrent largement la nouvelle pensée esthétique dans tout le Brésil. C'est ainsi que Luis Nunes, ancien élève de l'ENBA, réunit à Recife autour de lui un groupe de jeune ingénieurs, architectes et artistes parmi lesquels Roberto Burle Marx, qui allait créer en 1935-1937 son premier jardin pour la place Casa Forte. Citer dans ce contexte le nom de l'ingénieur Joaquim Cardoso, c'est rappeler combien le mouvement moderne s'est appuyé sur le travail d'équipe et à quel point les solutions techniques liées au fer et au béton étaient essentielles dans la conception même de cette architecture. Cardoso jouera d'ailleurs un rôle fondamental dans le développement des portées et des porte à faux de l'architecture de Oscar Niemeyer. 


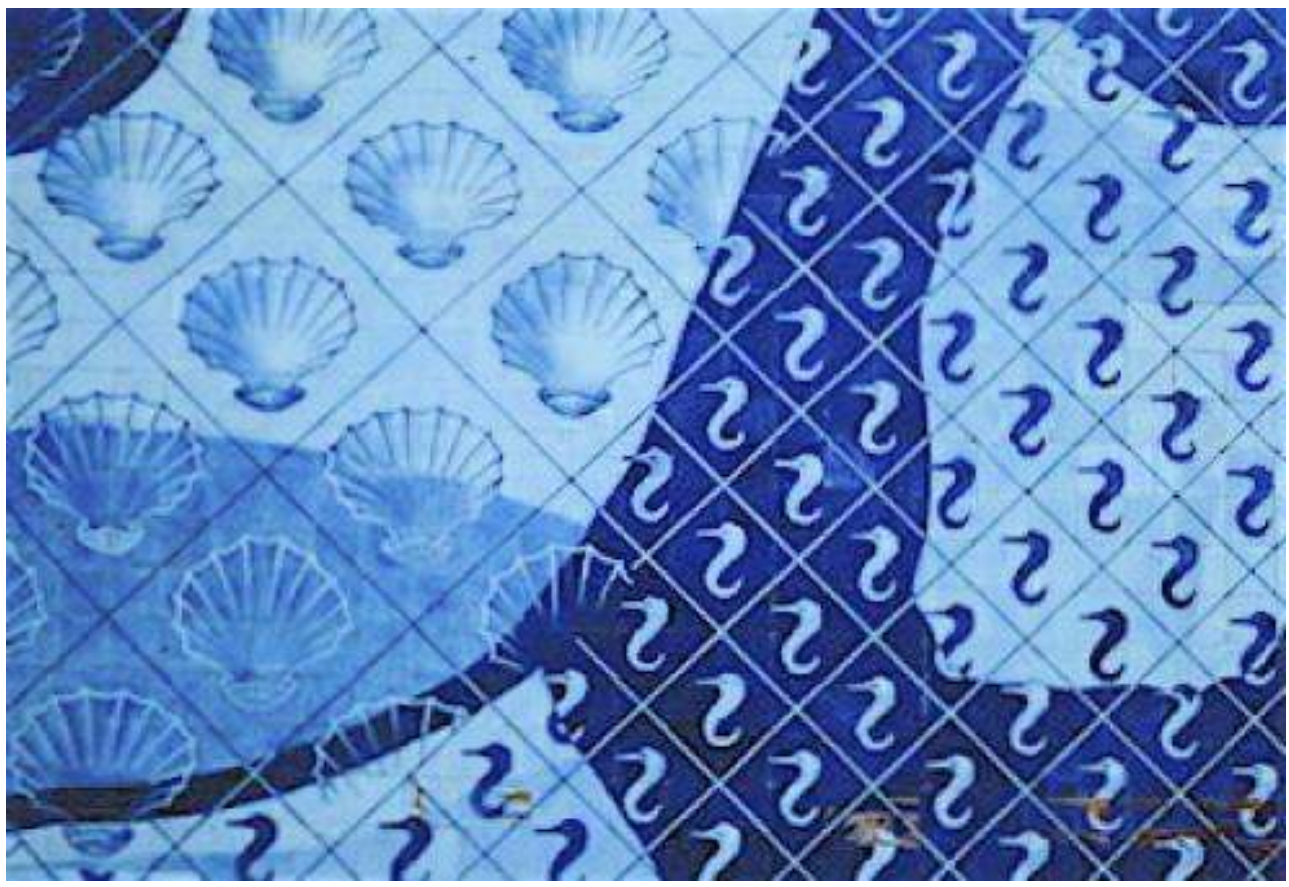

9 Le projet déposé par Lúcio Costa et son équipe pour le Ministère de l'Education et de la Santé avait prévu que Le Corbusier viendrait lui-même comme consultant de l'équipe brésilienne. Leur collaboration aboutit à une œuvre architecturalement exemplaire, marquée par l'intervention de l'artiste Cândido Portinari (azulejos) et par les jardins de Roberto Burle Marx, en terrasse et dans les espaces dégagés autour du bâtiment. De plus, le concept retenu apportait au cœur de l'urbanisme assez étouffant du centre de Rio de Janeiro un sentiment étonnamment fluide de l'espace. Les architectes avaient respecté le cahier des charges contraignant grâce à une construction très en hauteur qui ménageait de larges espaces libres à son pied, renforcés par l'usage de pylônes dégageant la vue sur les rues alentour.

10 Mais ce qui importe dans cette aventure trans-continentale, c'est que cette modernité si désirable constitue un puissant levier symbolique qui fait converger tous les peuples et toutes les cultures vers une forme unique d'habitat et bientôt de culture. L'homme unidimensionnel que nous sommes habitués à considérer aujourd'hui comme une aliénation insupportable est nimbée alors de l'auréole du progrès. Comme le dit avec force Michel Ragon : «Le temps de Le Corbusier est encore celui des Etats forts, de la montée des dictatures : Mussolini en 1922, Staline en 1924, Salazár en 1932, Hitler en 1933, Franco en 1938. Loin d'effrayer les intellectuels dont le rôle ne se justifie pourtant que par leur liberté d'esprit, ces dictatures les fascinent. (...) On ne saurait donc s'étonner de voir Le Corbusier vampirisé par la figure allégorique du Pouvoir. Il a dédié son livre La Cité radieuse " à l'autorité ". ${ }^{3}$

11 Il faut ajouter à la série des pouvoirs forts, l'Estado Novo de Getúlio Vargas qui, dans le moment même où il virait à la dictature, porta à son efficacité maximum la volonté de modernisation. C'est dans ce contexte extrêmement complexe que se développent les efforts de l'équipe de Costa pour promouvoir une architecture moderne, et dans ce cadre, donner une place au travail particulier de Burle Marx sur l'intégration de la végétation au bâti. 
Déjà, à l'époque de Lounatcharski, le constructivisme russe avait entrepris d'articuler les éléments plastiques et architecturaux dans une conception d'œuvre totale. Les arts décoratifs et l'architecture étaient appelés à collaborer pour le plus grand bonheur, esthétique autant que social, de la société nouvelle, avant que Staline ne siffle la fin de la partie. Le Bauhaus du début des années 30 , avant que Hitler ne disperse à son tour ses professeurs, avait repris le flambeau. Au Brésil, le développement du service et des bâtiments des Postes et Télécommunications illustre assez bien comment les principes d'un fonctionnalisme esthétique furent diffusés dans la conscience artistique et l'administration des établissements postaux. La Révolution de 1930, en mettant fin à la «República Velha » qui s'était construite sur les bases économiques du libéralisme, ouvrait les portes du pouvoir à de nouvelles couches sociales et faisait du dirigisme centralisé un nouveau principe de gouvernement.

Deux bureaux de poste réalisés à partir d'un même type architectural

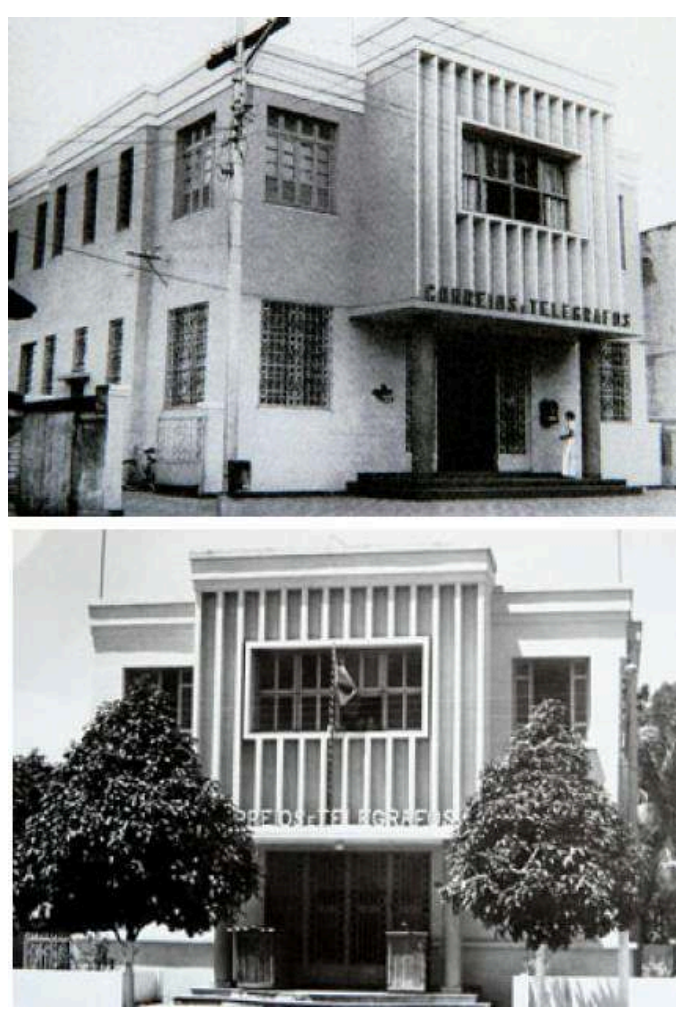

En haut à Araguari ; en bas à Macapá, voir Margaret da Silva Pereira, note 4 
Deux bureaux de poste réalisés à partir d'un même type architectural

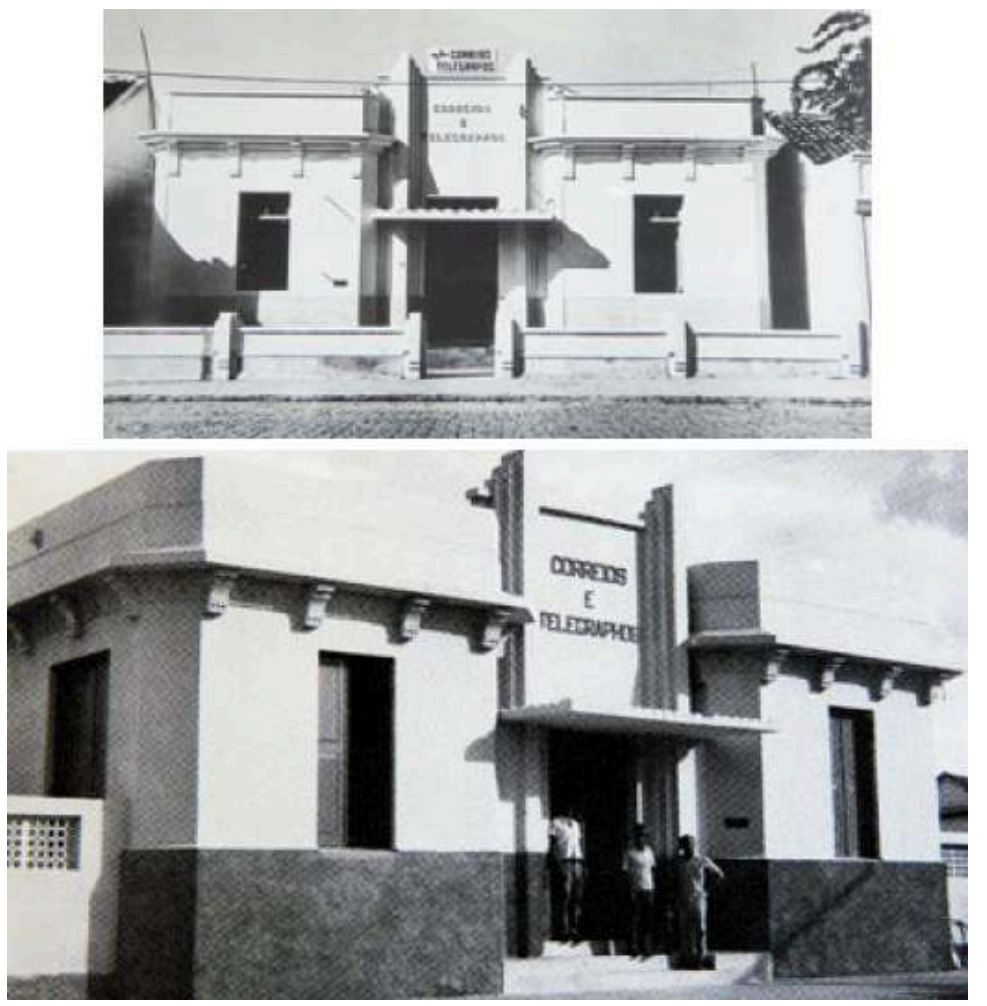

En haut à Quixeramobim CE ; en bas à Princesa Isabel PB, voir Margaret da Silva Pereira note 4

13 On avançait l'idée d'un service public et créait une administration centrale des postes et télégraphes, la DCT, jusqu'alors dispersés entre des mains privées ${ }^{4}$. Son programme de développement s'appuyait sur la standardisation des services et sur l'industrialisation de la construction des agences dont la vocation était de couvrir désormais tout le territoire national pour en désenclaver les régions les plus reculées. Et celles-ci ne manquaient pas dans un pays dont le développement industriel était très fortement concentrée dans le sud. On élabora donc quatre types de bureaux de postes correspondant à la taille des villes destinées à les recevoir, qui seraient répétés dans tout le pays. On commença par le Nordeste déshérité qui avait très fortement contribué à la « Révolution ». 


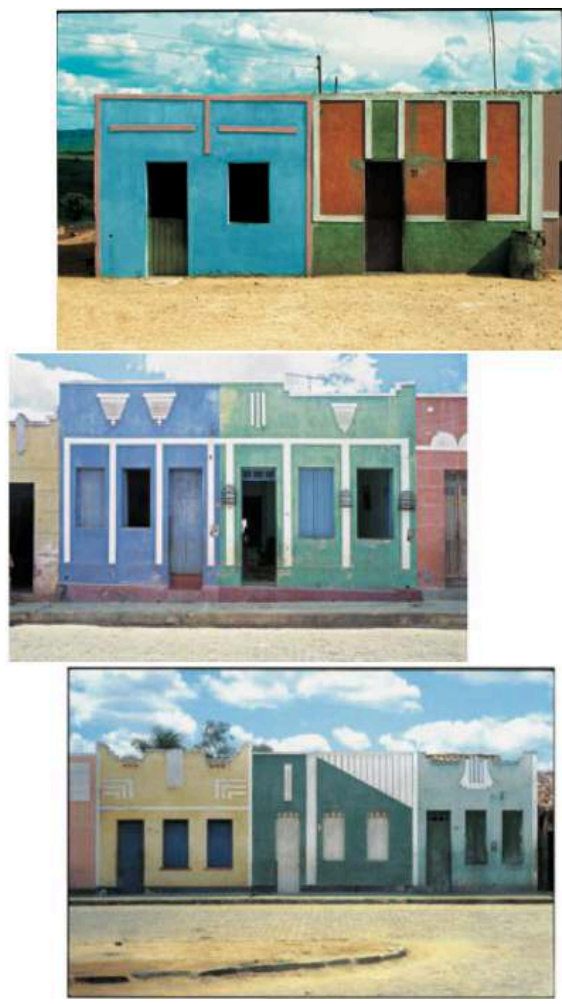

L'ensemble de ce travail de relevé photographique a été republié récemment dans son livre : Pinturas e Platibandas, IMS, Instituto Moreira Salles, São Paulo, 2010. publics Juarez Távora, allait diffuser le style art déco et les principes architecturaux modernistes dans tout le pays. Elle s'appuyait en outre sur le mouvement qui agitait depuis longtemps le monde des architectes à la recherche d'une pratique plus sociale de l'architecture. De plus, cette volonté allait de pair avec l'importance que prenaient les ingénieurs dans un mouvement de modernisation technique privilégiant en particulier l'usage du béton armé.

C'est dans ce sens qu'agit Lúcio Costa, en 1931, lors de son rapide passage à la tête de l'Ecole Nationale des Beaux-Arts, où il s'employa à rénover l'enseignement de l'architecture pour le libérer des contraintes liées à l'usage des matériaux traditionnels. De sorte que, en 1936, lorsqu'il fait appel à Le Corbusier, Lúcio Costa s'inscrit dans un mouvement, certes encore très controversé mais qui peut s'appuyer sur un désir de modernisation répandu dans de très vastes secteurs de la société.

position de Burle Marx dans ce dispositif est intéressante. Il a séjourné en Allemagne, à la fin des année 20, où l'enseignement du premier Bauhaus n'a pas pu le laisser indifférent. Il est cependant plus tourné vers la peinture et la musique que vers l'architecture. C'est Lúcio Costa qui l'oriente vers l'art des jardins en lui confiant son premier chantier : les espaces entourant la demeure d'Alfredo Schwartz à Copacabana (1933). Quelques années après, alors qu'il vient de réaliser trois jardins publics à Recife, Lúcio Costa lui propose l'aménagement des jardins qui doivent occuper le toit d'une partie du bâtiment du Ministère de l'Education et de la Santé et son environnement immédiat. 
17 Attardons-nous sur ce projet exemplaire. On perçoit immédiatement, à la vue du dessin qu'il produit pour la terrasse, l'importance que Burle Marx accorde à la composition des formes dans la surface qui lui est proposée. Le dessin gouaché préparatoire que nous possédons (1938) est dominé par des lignes courbes reliant des formes aux contours géométriques. Des superpositions de couleurs laissent imaginer la volumétrie ainsi que les jeux chromatiques que concrétiseront les plantes. La simplification des formes et les aplats colorés que constituent les plates-bandes constituent un effort très nouveau de rationalisation des formes dans le domaine du jardin, traditionnellement placé sous le signe de la variété botanique. Burle Marx met en œuvre une esthétique des formes simples. Son jardin oppose le végétal des plantations au minéral du bâti et les courbes à la domination de l'angle droit. En cela, Burle Marx reste proche de certains enseignements qui se donnaient au Bauhaus, comme celui de Kandinsky, dont le tableau Jaune, rouge, bleu. (1925) exploite très savamment le contraste des lignes et des jeux de superpositions.

Roberto Burle Marx. Plans colorés de la Résidence Odette Monteiro, Corréias, RJ

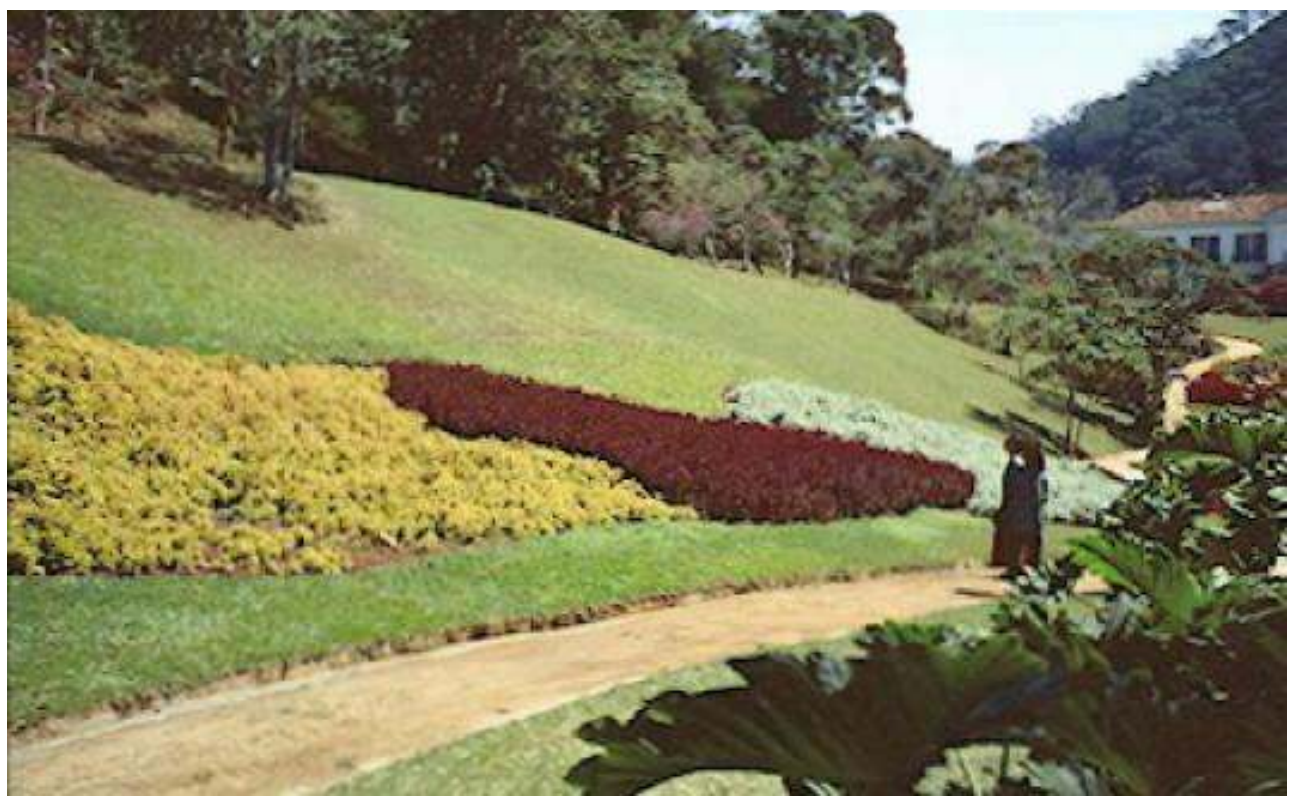

Le traitement en aplats des couleurs renvoie par ailleurs à l'enseignement que dispensait alors Fernand Léger, dans lequel ce dernier affirmait la valeur architectonique de la couleur. Dans les années 1923-1924, Léger abandonne en effet dans sa peinture la touche comme moyen pour rendre les volumes. L'effet de volume ne résulte plus d'un dégradé : il est produit par la franche césure d'un ton plus foncé, comme on le voit dans l'esquisse de Burle Marx. Alors que, dans les années de l'immédiat après-guerre, Ozenfant et Le Corbusier développent leur théorie puriste, l'aplat de couleur devient un des signes majeurs de la modernité picturale, publicitaire ou architecturale. Il fait partie de cet ensemble de principes esthétiques et architectoniques qui symbolisent les nouvelles valeurs de précision, d'ordonnance et de fonctionnalité qui animent, au Brésil, l'équipe réunie par Lúcio Costa. 
Roberto Burle Marx. Parc Roberto Burle Marx, São Paulo

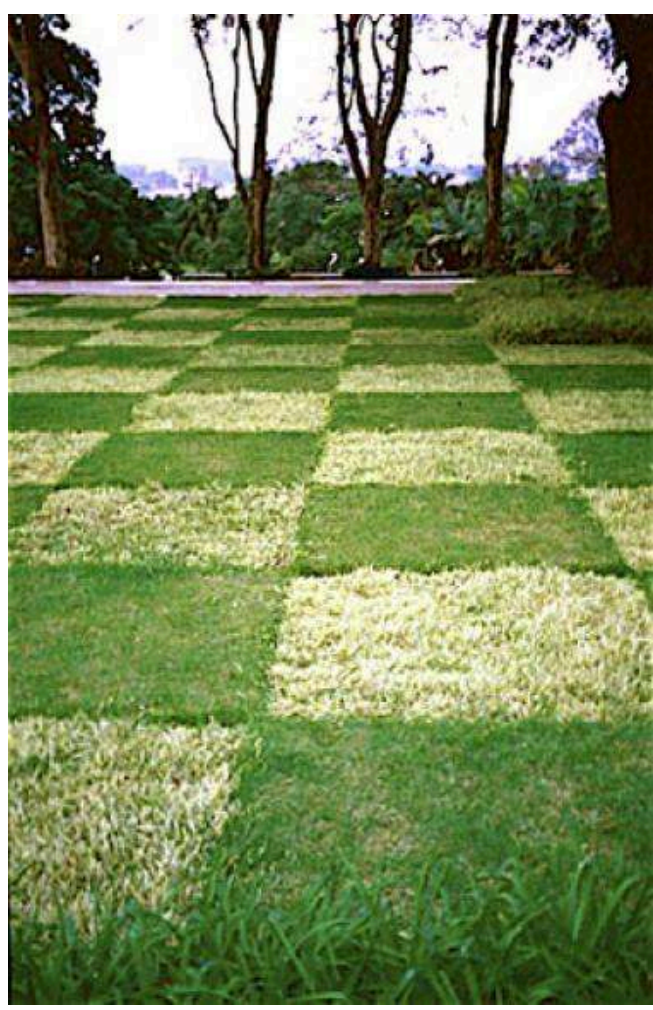

La période moderniste brésilienne est particulièrement intéressante dans la mesure où l'ouverture au langage universalisant de la géométrie se trouve constamment en tension avec le besoin d'affirmer des valeurs locales, destinées à favoriser l'émergence d'une conscience identitaire nationale. Tel était en tout cas l'horizon que proposait Oswald de Andrade en affirmant la nécessité d'un retour aux valeurs propres du pays. Il s'agissait d'alimenter l'élan moderniste aux sources vives des cultures sur lesquelles il s'était constitué : africaine, indienne et portugaise, sans oublier celles qui avaient été apportées au fil des dernières décennies par les nombreuses émigrations d'origine européennes. "Tupy or not Tupy", illustre sur le mode ironique, la double transcendance que Oswald de Andrade reconnaît aux apports culturels autochtones et étrangers.

En ce qui concerne l'urbanisme et l'art des jardins, ceux-ci étaient, de La Havane à Buenos Aires, fortement marqués par une série de figures obligées développées par divers disciples de la tradition haussmannienne. Les grands noms en furent: J.-Cl. Adolphe Alphand (1817-1891), Eugène Courtois (1843-1914), Charles Thays (1849-1934) et Jean-Claude Nicolas Forestier (1861-1930). Si la plupart se contentèrent d'exporter les modèles qu'ils avaient créés ou appliqués à Paris, Forestier, riche d'une expérience en Espagne et au Maroc, développa des jardins de plantes indigènes valorisant les beautés de la nature locale ${ }^{5}$. Il y avait eu en outre à Rio l'activité botaniste et paysagère de Auguste François Marie Glaziou (1828-1906) 6 qui avait, auprès de l'empereur Don Pedro II, puissamment contribué à la remise en valeur de la flore locale.

Burle Marx pressent dès le début que le nouveau jardin brésilien doit s'affranchir des modèles anglais et français dominants. Cette renaissance passe par la restauration dans ses droits légitimes de la flore autochtone dont il avait lui-même découvert à la fin des années 20 les splendeurs, là-bas exotiques, dans les serres du jardin botanique de 
Berlin. C'est pourquoi la question de la botanique prend chez lui, dès le départ, une importance particulière dans son œuvre. Il écrit : 'Sauver au moins une petite partie de notre flore disséminée, récolter des spécimens dans la nature, découvrir leur potentiel esthétique pour le paysagiste, multiplier les espèces afin de pouvoir les faire convenablement figurer dans les jardins, démontrer leur grande valeur si elles sont correctement utilisées, en harmonie avec le milieu, devint ma tâche comme paysagiste'.

Sítio, serres dans lesquelles Burle Marx faisait reproduire les espèces végétales qu'il utilisait pour ses créations de jardins

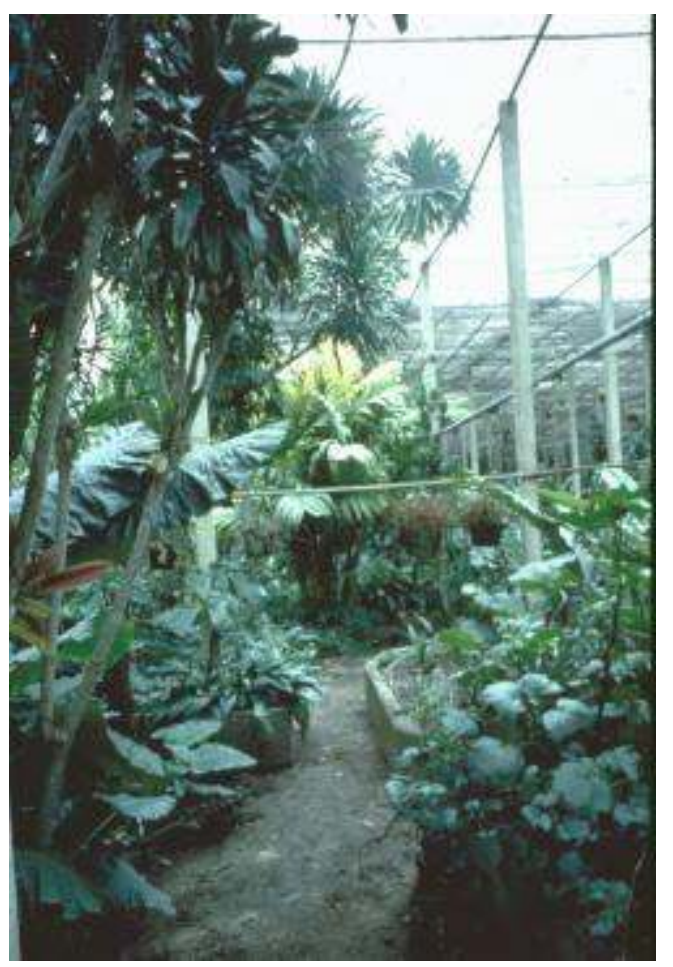

Cependant la collecte de spécimens et plus encore la reproduction en serres des plantes collectées, pourrait conduire à cette abstraction qui fait de la plante un objet abstrait qu'on manipule, une entité artificielle séparée de son biotope. C'est pourquoi la collecte fut pour Burle Marx à la fois une école et une éthique, discipline personnelle et responsable, à laquelle il s'est astreint sa vie durant.

De cette expérience sur le terrain Burle Marx retirera un enseignent essentiel concernant la socialité des plantes. Des nombreux voyages de collecte qu'il entreprend accompagné de divers botanistes, Brade d'abord, à partir de Recife, dans la Serra do mar, où il se familiarise avec la végétation des territoires très secs de la caatinga, puis Henrique Mello Barreto, Burle Marx apprend la relation précise qu'entretient la plante avec son biotope, et à l'intérieur de celui-ci, avec les autres plantes et les autres êtres vivants avec lesquelles elle vit véritablement en association. Il insistera toujours sur sa dette à l'égard de Mello Barreto. C'est en effet en sa compagnie qu'en 1943 il entreprend la réalisation du Parc d'Araxa, dans le Minas Gerais. Le botaniste lui fait étudier une à une, systématiquement, les associations de plantes se développant dans les différents types de terrains: calcaires, gneiss-granitiques, basaltiques etc. «Franchissant les frontières de l'activité paysagiste, à savoir la simple composition esthétique du jardin, je commençai à comprendre l'importance que revêt la mise en 
valeur de notre flore, celle des contraintes environnementales de chaque plante, les associations végétales se transformant elles-mêmes en ensembles esthétiquement et biologiquement équilibrés ${ }^{8}$ Ce travail débouchera sur des principes paysagistes absolument spécifiques du fait même de cette relation à la flore latino-américaine : «Durant ces années de travail en commun avec Mello Barreto, nous avons réalisé ensemble divers projets dans lesquels les concepts paysagers et écologiques se complétaient dans notre effort pour faire connaître ces formations propres à notre terre. $»^{9}$

En donnant pour des raisons locales spécifiques son plein sens à l'idée de jardin écologique, Burle Marx inaugure une nouvelle époque de l'art du jardin latino-américain, et, d'une manière générale, de l'art contemporain du jardin. Il quitte petit à petit la modernité formelle du début du siècle pour construire les bases d'une nouvelle modernité, celle qui se placera sous les exigences des disciplines écologiques. Ces nouvelles perspectives et leurs implications théoriques et formelles ne changent toutefois rien à cette autre dimension de la pensée moderne : la volonté de faire, à travers l'art, œuvre de conviction et de formation. Burle Marx a toujours souligné la forte volonté qui l'animait de faire de ses jardins des instruments de plaisir et de pédagogie, sans que l'un de ces aspects entre en contradiction avec l'autre.

Sítio, associations végétales en partie basse et humide

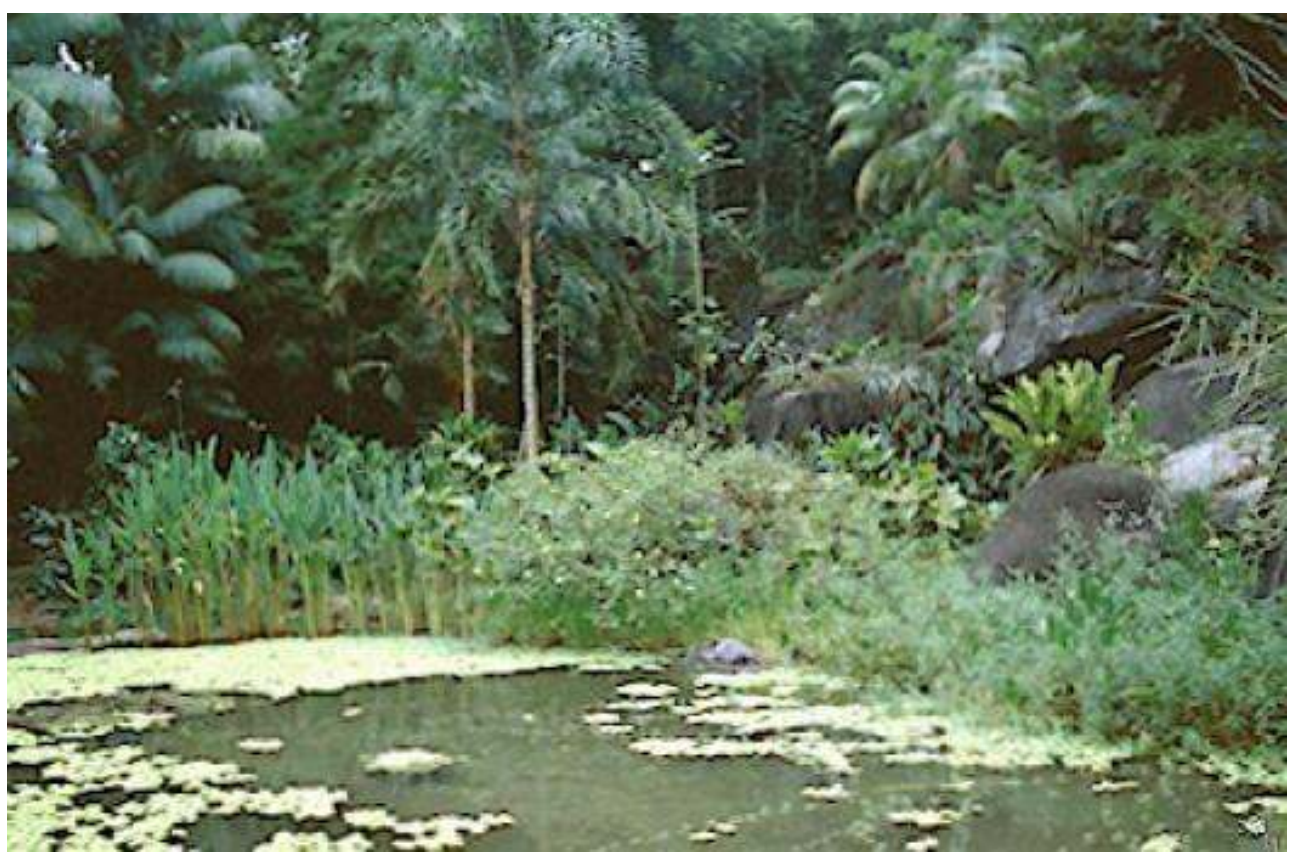

Dans un article sur 'Les jardins géographiques, lieux et espaces de mémoire', ${ }^{10}$ Jean-Marc Besse rappelle la pratique, éparse aux XVIIe et XVIIIe siècles, de concevoir des jardins comme des instruments pédagogiques au service de la géographie. Il s'agissait, en réalisant une métaphore matérielle de l'espace concret d'un pays à échelle réduite, de donner à voir la géographie, mieux même, de la donner à sentir. Cette science, trop abstraite, laisse en effet le spécialiste devant la tâche insurmontable de faire comprendre, à travers des concepts topographiques et des descriptions, un objet éminemment sensible. En articulant le territoire réel, le jardin comme microcosme, et la carte comme espace conceptuel, le jardin géographique crée les conditions d'une 
expérience complexe dont l'instabilité même, liée aux changements constants d'échelle, est porteuse d'un pouvoir puissant sur l'imagination.

Sítio, cactées de la zones haute et sèche

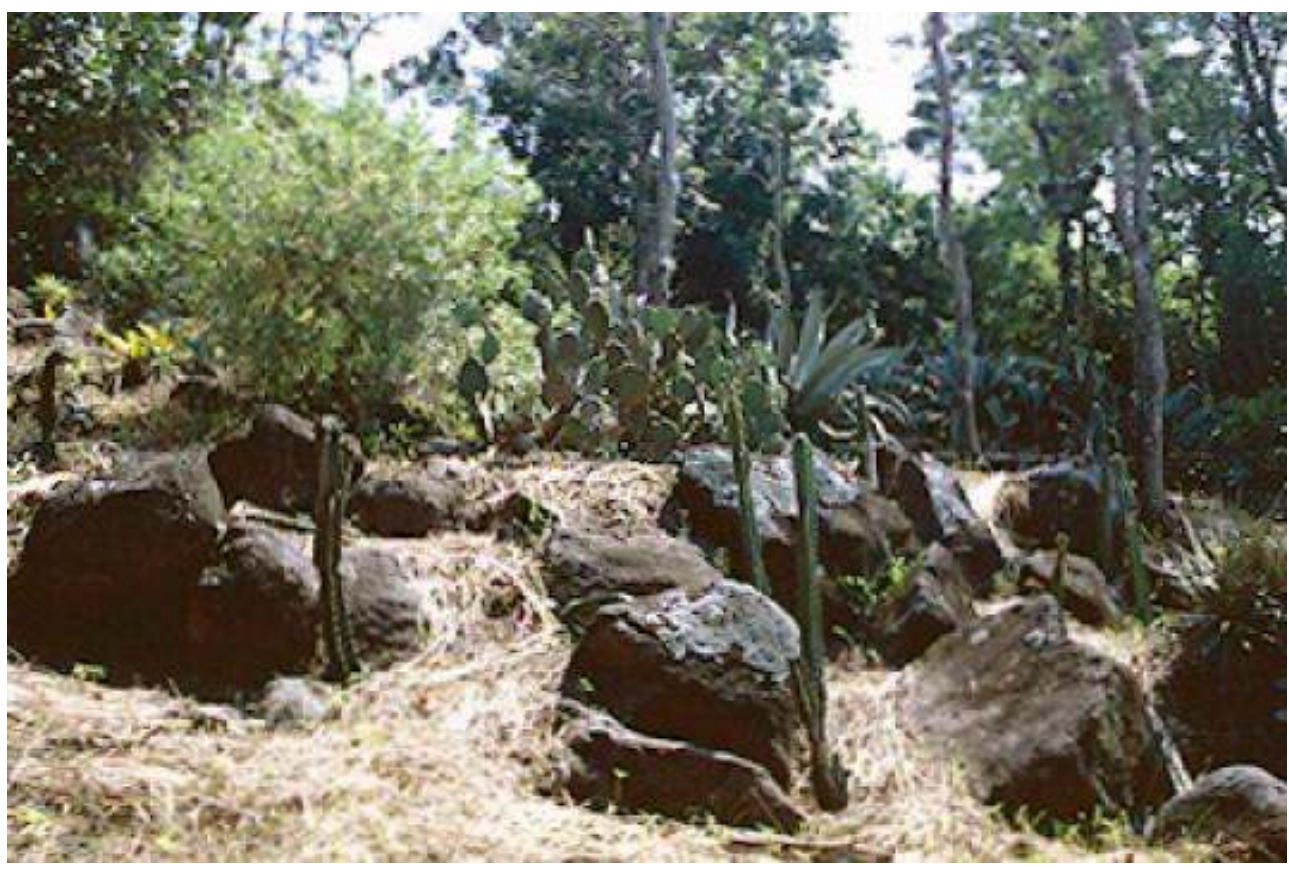

26 Le jardin que Burle Marx établit avec sa résidence et ses serres à San Antonio da Bica, le Sitio n'est pas sans rapport avec cette volonté pédagogique à l'égard de la conscience et de la mémoire nationales. Il ne s'agit pas, comme dans les projets destinés aux princes et aux rois auxquels se réfère Besse, de mimer en réduction la géographie en ses territoires, en représentant par exemple une forêt avec des herbes, mais bien plutôt de créer sur une aire réduite, un microcosme du Brésil et de sa flore à des fins à la fois esthétiques et pédagogiques, selon une procédure instructive parce que révélatrice de beautés.

27 Ce jardin a été établi en un lieu offrant des possibilités d'acclimatation pour toutes les espèces autochtones de la flore brésilienne, ce qui implique évidemment des conditions physiques très diverses. Le Sitio a donc été choisi, puis aménagé, de manière à offrir un couvert suffisamment dense pour des plantes telles les philodendrons, dont plus de 400 espèces y ont été installées. De même les begonias, orchidées et toutes les espèces de broméliacées qu'affectionne particulièrement Burle Marx, y trouvent leurs conditions d'existence, sans parler des nombreuses plantes épiphytes si caractéristiques de la forêt amazonienne. La géologie locale permettait en outre de fournir un environnement favorable aux espèces rupestres pouvant se développer au voisinage des blocs granitiques dispersés sur le terrain.

28 Enfin Burle Marx aménagea une zone humide où la flore des régions de l'Amazonie, de Goiás et de Bahia telle la célèbre Victoria Regia et diverses espèces de nymphéas trouvèrent un milieu de subsistance adéquat. Ainsi, le jardin du Sítio es-il dessiné non pas en fonction d'une recherche de formes mais selon la logique des climats et de la géologie propres aux différentes régions du Brésil.

29 C'est ainsi que Burle Marx met en évidence sa conviction que le motif moderne par excellence n'est pas, comme le laisserait entendre une conception bornée, la réduction 
de la nature à l'artifice, mais la reconnaissance des organisations complexes qui réunissent les plantes dans des écosystèmes extrêmement élaborés.

Les transferts de modèles esthétiques ne constituent jamais des procédures simples. Un modèle n'a tout son sens que sur la terre et dans la culture qui l'ont produit et l'ont vu naître. Ainsi, on s'illusionne en pensant que les formes que l'on croit retrouver sous d'autres cieux constituent de simples copies de celles auxquelles notre savoir les associe et où il voit leur origine. En réalité, plutôt que simplement transférées, elles ont été reprises, re-symbolisées et donc en quelque sorte recréées et chargées de nouvelles significations. Filles adultérines d'un modèle lointain, elles vivent une vie autonome là où elles ont été acclimatées. Sont-elles encore les mêmes ou sont-elles devenues autres ? La dialectique des échanges mondialisés, qui s'est accélérées depuis plusieurs décennies, enseigne la prudence lorsqu'on croit pouvoir parler d'influence. L'exemple de la créativité brésilienne est là, une fois encore, pour le démontrer.

\section{NOTES}

1. Mario de Andrade, "Modernismo e Ação ", publié dans le Jornal do Commercio du 24/5/1925, reproduit in Jorge Schwartz, Vanguardas latino-americanas, Polêmicas, manifestos e textos críticos, Edusp, São Paolo 1995, p. 477

2. voir: Carlos A. Ferreira Martins, "Construir uma arquitetura, construir um pais » in Da antropofagia a Brasília, Brasil 1920-1959, Jorge Schwartz organisateur, catalogue de l'exposition éponyme au MAB-FAAP, São Paulo, Cosac\&Naify 2002, pp. 373-383

3. Le temps de Le Corbusier, sous la direction de Michel Ragon, Hermé, Paris, 1987 p. 20

4. cf. Margareth da Silva Pereira, Os correios e telégrafos no Brasil, Um patrimonio histórico e arquitetônico. Empresa Brasileira de Correios e Telégrafos, Brasilia,1999, p. 123 à 175

5. Salvador Tarrago I Cid «Entre Le Nôtre y Le Corbusier », in J.Cl. Nicolas Forestier : Du jardin au paysage urbain, Picard, Paris, 1994, p. 255

6. voir le catalogue de l'exposition Glaziou e os jardins sinuosos, Jardin botanique de Rio de Janeiro, novembre 2009-janvier 2010

7. Roberto Burle Marx, Arte e paisagem, São Paulo, Nobel, 1987 p. 48

8. idem p. 83

9. idem p. 50

10. Jean-Marc Besse, Le jardin, art et lieu de mémoire, Besançon, Editions de l'imprimeur, 1995

\section{RÉSUMÉS}

Burle Marx met en évidence sa conviction que le motif moderne par excellence n'est pas, comme le laisserait entendre une conception bornée, la réduction de la nature à l'artifice, mais la 
reconnaissance des organisations complexes qui réunissent les plantes dans des écosystèmes extrêmement élaborés.

INDEX

Mots-clés : Burle Marx (Roberto), Niemeyer (Oscar), architecture, jardin géographique, Sitio

\section{AUTEUR}

JACQUES LEENHARDT

Ecole des Hautes Etudes en Sciences Sociales 